\title{
Mental health in primary healthcare in Chile
}

\author{
Alfredo Pemjean
}

Department of Mental Health, Ministry of Health of Chile; Professor of Psychiatry and Mental Health, Universidad Diego Portales, Santiago, Chile, email alfredopemjean@vtr.net

\section{Caser} hile has two major national health systems, the public one, which serves nearly $80 \%$ of the country's close to 17 million population, and the private one, which serves the other $20 \%$. The public primary healthcare system has been developing in Chile since before the Alma Ata Conference in 1978 (which produced the first international declaration on the importance of primary healthcare).

There are local out-patient general health centres throughout the country. The system has been designed as a network, comprising larger and smaller units: the larger units are the 294 family health centres and 257 general dispensaries, which are intended to serve localities with 20000-30000 inhabitants, although several in big cities provide care for populations of up to 60000 , while others serve fewer than 6000; the smaller units are the more than 1500 rural health clinics, which serve localities with 150-2500 people.

In the early 1960s, health centres were staffed by four professionals in a general health team (general practitioner, nurse, midwife and social worker). Since then, the range of expertise has increased and diversified, particularly in the past 10 years. Nowadays, it is usual to find physiotherapists, dentists, nutritionists and psychologists working at these centres, especially in urban areas.

\section{Primary mental healthcare}

In Chile, the recent health administrations have given high priority to supporting primary health centres, with the intention that they respond to a wider variety of health needs, including mental health (Ministerio de Salud, 2000). Between 2003 and 2008, at the national level, the number of fulltime medical jobs in the centres increased by $146 \%$, and the number of full-time psychologists increased by $344 \%$.

Early in the 1990s, several mental health surveys gave cause for concern about the high proportion of users of primary healthcare facilities who were affected by mental health problems (Araya et al, 1994, 2001; Ustun \& Sartorius, 1995; Florenzano et al, 1998). As a consequence, primary health expanded the definition of its role to include mental healthcare, covering anxiety and depressive symptoms and disorders, psychosocial problems (such as pregnancy among adolescents), family violence, child abuse and drug misuse. This initiative facilitated a rapidly growing provision of training, leading to gains in experience and self-confidence among general health teams in the management and treatment of patients with psychosocial and psychiatric problems.

Today, one or more general practitioners, with variable levels of mental health training, participate in mental health teams within every urban primary health centre. They work jointly with psychologists and social workers, receiving referrals from other professionals at the same centre, and act as a first level of screening, making preliminary diagnostic and treatment decisions.

Some of the tools they use include the 'integral diagnosis session', which is a half-hour interview by three professionals. This is much longer than the usual 12-minute session the patient would receive from a general health attendant. After this initial session, the professional team comes to a consensual decision on the best treatment plan for the patient, which may include medical and psychological input by appropriately trained professionals. There may be: individual or group psychosocial interventions; individual or group psychotherapy undertaken by psychologists; and home visits and interviews by social workers, nurses, midwives, or others.

\section{Interface between primary and secondary care}

In the 'mental health consultation' form of assessment, the patient is the subject of a discussion between two or three specialists, who make regular visits to the primary care centre for the purpose of having a clinical meeting on difficult clinical cases. Sometimes this happens in the presence of the patient; on other occasions there may be a review of a set of clinical records and, sometimes, a meeting on technical or administrative issues. This is the inverse of the patient going to the specialist, which was the usual route in the health system before the introduction of this new arrangement.

By this means, a common space for discussion by generalists and specialists is gradually being created in order to meet patients' individual needs, and it should lead to an increase in treatment adherence, by reducing dropouts in the transitional phase between initial consultation and the implementation of therapy.

There are over 60 community mental health centres, which work closely with the primary healthcare centres, a system that has been evolving over the past 15 years. Staff in the two types of institution in many cases cooperate in the overall planning process for mental healthcare, and they are in frequent contact. Community mental health centres will become the main means of delivering secondary-level psychiatric care in the country in the near future.

In 2004, a plan for health reform defined a protected pathway of care for 56 illnesses, which assures access, opportunity, quality and financial support to all people, in 
both public and private health systems (an 'explicit health guarantees system'). The choice of illnesses covered by this scheme was made on the basis that they involved a major burden of disease, that there was evidence-based treatment available, and that it was feasible for the health system to cope with them. Mental health problems included in this scheme are: schizophrenia, from the first episode (with both early detection and continuity of care); depression; and alcohol and drug misuse in people under 20 years of age.

In the management of these conditions, the primary health centres play a crucial role. They provide early detection ('diagnosis suspicion') for all of them. They also provide treatment according to clear clinical guidelines (Ministerio de Salud, $2005,2006,2007)$. Treatment of less severe cases is specified in a primary care protocol in some instances. However, if there are signs of bipolar disorder, suicide risk, violent behaviour, psychosis or severe comorbidity, the arrangements require referral to specialty care. Secondary referral is also required if treatment in the primary care centre has failed over a defined period.

This policy has meant that mental healthcare is a key aspect of the identity of primary healthcare in Chile, and such services now provide care to more than $80 \%$ of the 500000 people who are receiving mental healthcare in the whole of the public health system.

\section{Weaknesses of the system}

There are some weak components of mental health management within the primary care system. First, adherence to treatment, follow-up and clinical outcome indicators are not evaluated adequately; there is no system for recording them in the regular administrative procedures of the health system. This deficiency represents a serious gap, which hinders a reliable estimate of the outcomes and quality of care. In the case of depression, audits which were done up to 5 years ago found that, of the $30 \%$ of patients remaining in treatment 6 months after entering the depression programme in primary healthcare centres (mainly moderate and severe cases), between $7 \%$ and $10 \%$ had subsequently been referred to specialists, and $7 \%$ had been discharged. A significant improvement in symptoms, with patient satisfaction, was found in those receiving both pharmacological and/or psychosocial interventions (Alvarado et al, 2005). Within the national public health network there is a complex system for monitoring the course of all the diseases treated under the health plan; this allows professionals to know, for instance, how many people with depression are under treatment, or waiting for treatment, within or outside of the scheduled waiting time (guarantee of opportunity). However, crucial data such as outcomes (including partial recovery, relapses and critical events) are not yet being recorded comprehensively.

Second, community participation is scarce, even now. Communities do not have enough influence in the planning decisions of health authorities, local or national. In this way, cultural and social resources cannot contribute to these processes, which is a loss because they could be a valuable complement to health system activities. For example, community self-help groups could provide support to those who are misusing alcohol and drugs or who suffer from depression and anxiety. Suggestions of this nature rarely evoke a positive response from the professionals involved, and there is often no clear procedure by which such groups could work together with primary healthcare professionals.

Third, clinical academic teaching and research remain remote from the change in the nature of primary care. Except for some isolated examples, the undergraduate medical teaching and psychiatric specialty training programmes continue to be focused on a biomedical approach to medicine and on psychopharmacological treatments. They disregard the value of taking an interdisciplinary approach to the treatment of mental health disorders and ignore the community mental health model, even though it is explicitly defined to be the foundation of the Mental Health and Psychiatry National Plan.

\section{References}

Alvarado, R., Vega, J., Sanhueza, G., et al (2005) Evaluacióndel programa para la Detección, Diagnóstico y Tratamiento Integral de la Depresión en la Atención Primaria en Chile. [Evaluation of the Programme for Depression Detection, Diagnosis, and Comprehensive Treatment in primary care in Chile.] Revista Panamericana de Salud Pública, 18, 278-286.

Araya, R., Wynn, R., Leonard, R., et al (1994) Psychiatric morbidity in primary health care in Santiago, Chile. Preliminary findings. British Journal of Psychiatry, 165, 530-533.

Araya, R., Lewis, G. H., Rojas, G., et al (2001) 'Patient knows best' - detection of common mental disorders in Santiago, Chile: cross-sectional study. BMJ, 322, 79-81.

Florenzano, R., Acuña, J., Fullerton, C., et al (1998) Estudio comparativo de frecuencia y características de los trastornos emocionales en pacientes que consultan en el nivel primario de atención en Santiago de Chile. [Comparative study of the frequency and features of emotional disturbances in patients in primary care clinics.] Revista Médica de Chile, 126, 397-405.

Ministerio de Salud (2000) Plan Nacional de Salud Mental y Psiquiatría. Available at http://www.minsal.cl (accessed October 2009).

Ministerio de Salud (2005) Guia Clínica. Primer Episodio Esquizofrenia. [Clinical Guide. First Episode Schizophrenia.] Available at http:// www.redsalud.gov.cl/archivos/guiasges/EsquizofreniaR_Mayo10.pdf (accessed October 2009).

Ministerio de Salud (2006) Guía Clínica. Tratamiento de Personas con Depresión. [Clinical Guide. Treatment of Persons with Depression.] Available at http://www.redsalud.gov.cl/archivos/guiasges/depresion. pdf (accessed October 2009).

Ministerio de Salud (2007) Guía Clínica. Consumo Perjudicial y Dependencia de Alcohol y Drogas en Menores de 20 Años. [Clinical Guide. Detrimental Consumption of and Dependency on Alcohol and Drugs in Minors Under 20 Years.] Available at http:// www.redsalud.gov.cl/ archivos/guiasges/GPCGes-OHyDrogas-2007-rev26junio.pdf (accessed October 2009).

Ustun, B. \& Sartorius, N. (1995) Mental Illness in General Health Care: WHO International Study. Wiley.

As you pass from the tender years of youth into harsh and embittered manhood, make sure you take with you on your journey all human emotions! Don't leave them on the road, for you will not pick them afterwards! 